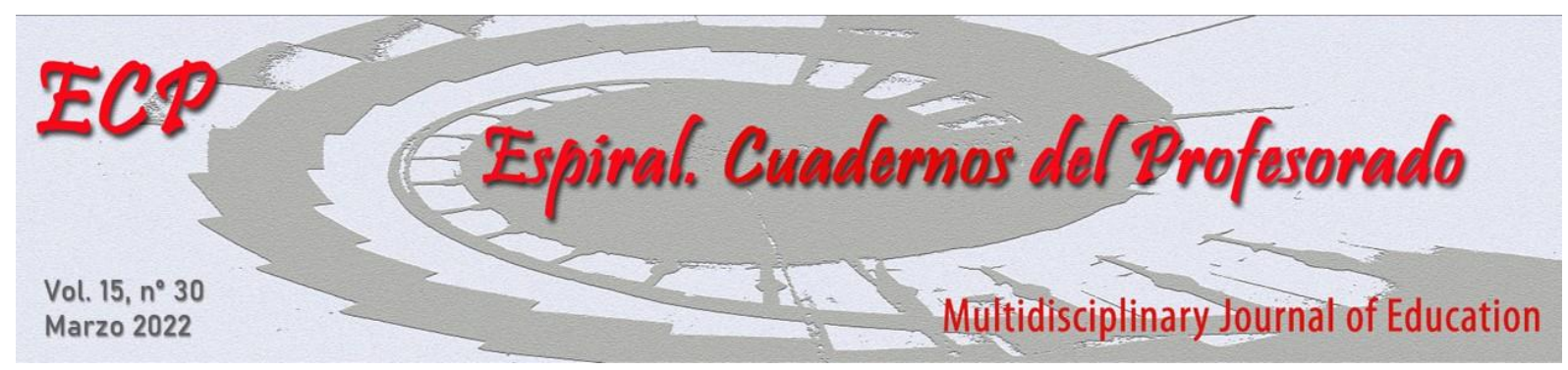

\title{
Actitudes docentes hacia alumnos con discapacidad, punto de partida para la educación inclusiva: una perspectiva de sexo, edad y experiencia previa.
}

\section{Teaching attitudes towards students with disabilities, basis for inclusive education: a perspective of sex, age and previous experience}

\author{
Patricia Solís García y Delia Arroyo Resino \\ Universidad Internacional de La Rioja, España
}

\begin{abstract}
Resumen
La educación inclusiva, especialmente en el caso de alumnos con discapacidad, está mediada por las actitudes de los docentes hacia este colectivo y hacia la propia inclusión. Partiendo de ello, el objetivo del presente estudio es analizar las actitudes docentes (acorde al sexo, edad y la experiencia) para conseguir una mejor comprensión de las necesidades actuales del sistema educativo. De este modo se analizan los datos sobre actitudes de una muestra de 281 docentes que desarrollan su función en diferentes etapas educativas (preescolar, primaria y educación secundaria), tanto en colegios públicos como privados, a lo largo de todo el territorio español. Se ha utilizado el cuestionario validado de actitudes de los docentes hacia los alumnos con necesidades educativas especiales, derivadas de la discapacidad, en su versión adaptada formada por 22 ítems que abordan: Responsabilidad, Rendimiento, Formación y recursos, Clima del aula y Desarrollo Social. En definitiva, los resultados arrojan actitudes moderadamente positivas hacia la inclusión. En general, los hombres presentan rangos promedios superiores a los de las mujeres, los docentes menores de 41 años puntúan más en la dimensión desarrollo social y clima del aula y tienen una mejor actitud los docentes que no presentan experiencia con estudiantes con discapacidad.
\end{abstract}

Palabras clave: Deficiencia; Actitud; Docentes; Inclusión educativa

\begin{abstract}
Inclusive education, especially in the case of students with disabilities, is mediated by teachers' attitudes towards this group of students and towards inclusion itself. The objective of this study is to analyze teaching attitudes to achieve a better understanding of the current needs of the educational system. In this way, the data on the attitudes of a sample of 281 teachers (69\% women) who perform their function in the different educational stages (preschool education $=18.1 \%$, primary education $=45.6 \%$ and secondary education $=36$ ) are analyzed, $3 \%$ ), both in public schools $(57.7 \%)$ and private $(42.3 \%)$ throughout the Spanish territory. The adapted questionnaire for teachers of attitudes towards students with special educational needs derived from disability has been used, made up of 22 items that address five dimensions: Responsibility, Performance, Training and resources, Classroom Climate and Social Development. In general terms, the results show moderately positive attitudes towards the inclusion of students with disabilities, studying differences according to sex, age and previous experience with students with disabilities.
\end{abstract}

Keywords: Disability; Attitude; Teachers; Inclusive education.

Fecha de recepción: 29/01/2021

Fecha de aceptación: 21/07/2021 


\section{Introducción}

Los modelos educativos responden a las necesidades de la sociedad y por ello se desarrollan y evolucionan conforme a los cambios de esta. Uno de los principales retos actuales es acoger y atender adecuadamente a la diversidad de la sociedad actual que se refleja en las aulas. Dicha diversidad comprende también al colectivo formado por personas con discapacidad, donde el modelo social identifica la discapacidad como un fenómeno cultural e históricamente específico en el que las personas con discapacidad son un grupo oprimido (Shakespeare, 2006). Por este motivo, "en las últimas décadas se ha venido impulsando una mayor sensibilidad hacia la discapacidad en sus diferentes manifestaciones, tanto desde las instancias internacionales como de las nacionales" (Rodríguez, 2015, p.224). La educación inclusiva de estudiantes con discapacidades en contextos de educación general ha sido un movimiento global durante al menos 30 años (Amor et al., 2019), calificado como el mayor desafío que deben enfrentar los sistemas educativos de todo el mundo (Ainscow, 2005). A pesar de las leyes y políticas, el progreso hacia una educación inclusiva que promueva el acceso significativo y el avance en el plan de estudios de educación general ha continuado despacio (Hagiwara et al., 2019), se están dando pasos hacia este modelo, pero no resultan sencillos, se trata de un camino largo (Garzón et al., 2016) en el cual la inclusión real se ve obstaculizada por determinadas condiciones (Pérez-Jorge et al., 2016).

Cuando hablamos de modelo inclusivo y educación inclusiva, no parece existir una única definición compartida (Pérez-Jorge et al., 2016) pero el elemento común es que el sistema educativo debe ajustarse para satisfacer las necesidades de todos los niños/as y jóvenes (Lindqvist, 1994). Para ello se requieren modificaciones y transformaciones de las estrategias y estructuras, con el fin de adecuarse a la diversidad del alumnado atendiendo a sus necesidades específicas y creando un espacio adecuado que pueda ser compartido por todo el alumnado (OCDE, 2013; UNESCO, 2009).

Si bien se han hecho muchas distinciones entre educación "especial" e "inclusiva" y se sigue debatiendo enérgicamente, los dos conceptos continúan siendo muy evidentes en la política y la práctica de muchos países (Florian, 2019), perpetuándose la inclusión con prácticas integradoras (Castillo, 2019). El paradigma de la educación especial todavía se basa en gran medida en el modelo médico de discapacidad que considera a la persona como enferma a la que hay que rehabilitar (Hall, 2018). No obstante, como ya hemos apuntado previamente nos encontramos inmersos en un modelo biopsicosocial de comprensión de la discapacidad. Interesa pues saber, cuáles son las dificultades a la hora de implementar un modelo educativo inclusivo. Aunque el progreso general en la educación inclusiva está vinculado al desarrollo de valores culturales, la promesa de una mayor inclusión en las escuelas va de la mano de la disponibilidad de recursos adecuados (Saloviita, 2019). Además de recursos, este proceso complejo requiere considerar las actitudes de toda la comunidad educativa, concretamente las actitudes del profesorado son fundamentales para atender a la diversidad del alumnado (Garzón et al., 2016). Estas actitudes docentes han sido reconocidas como un indicador clave para la inclusión adecuada de los estudiantes (Garrad et al., 2019), y son un requisito previo para una exitosa implementación (Saloviita, 2019).

El estudio de las actitudes hacia las personas con discapacidad ha ido cobrando interés y relevancia (Rodríguez, 2015). La primera definición de este concepto la encontramos en Allport (1935) quien se refiere a esta como "un estado de predisposición mental o neuronal, organizado a través de la experiencia, que ejerce una influencia directiva o sobre la respuesta del individuo a todos los objetos y situaciones con los que está relacionada" (p. 810). Tres décadas más tarde, Fishbein (1967) afirma que se trata de una predisposición aprendida que se orienta a la respuesta consistente a un objeto. Ha habido otra línea de interés centrada en el componente emocional, según la cual la emoción ocupa un rol vital para impulsar la acción ante ciertas circunstancias sociales, las actitudes son por tanto construcciones cognitivas complejas (Triandis, 1971). Podemos definir las actitudes como aquellas valoraciones que la persona hace sobre los objetos o los estímulos de su entorno social que implican maneras más o menos permanentes de pensar, sentir y/o actuar ante ellos. Refiriéndonos al colectivo que nos atañe, las actitudes deben entenderse como una forma de barrera inmaterial que limita la plena inclusión social y laboral y restringe la participación de las personas con discapacidad (Rodríguez, 2015). 
La investigación ha enfatizado consistentemente que los docentes son importantes para determinar el éxito de la implementación de la educación inclusiva (Kurniawati et al., 2017). Se espera que estos hagan frente a los estudiantes con diversas necesidades aun siendo posible que no siempre estén listos o que no tengan el apoyo suficiente para enfrentar estos desafíos (Gal et al., 2010). Las actitudes de los docentes hacia la inclusión a menudo se basan en la implementación práctica de la educación inclusiva más que en una ideología específica y comprensión de la inclusión (Vaz et al., 2015). Encontramos en los últimos años numerosos estudios que abordan en profundidad las actitudes de los docentes hacia la integración e inclusión del alumnado con discapacidad tanto en el ámbito nacional (Cruz-Ortiz et al., 2016; Garzón et al., 2016; Granada et al., 2013; Pérez-Jorge et al., 2016; Rodríguez, 2015) como internacional (Cagran y Schmidt, 2011; Dias \& Cadime, 2016; Garrad et al., 2019; Gal et al., 2010; Hind et al., 2019; Vaz et al., 2015). En el estudio de las variables sociodemográficas y su relación con las actitudes ha sido de interés: la influencia de la edad, el sexo y la experiencia como docente (Vaz et al., 2015). Así se ha explorado cómo influye el sexo del docente en las actitudes encontrando resultados contradictorios, aunque cuando se han hallado diferencias han sido las mujeres quienes mostraban una actitud más positiva (Solís y Borja, 2021; Vaz et al., 2015). Asimismo, se ha puesto el centro de interés en el estudio de la experiencia docente, aunque los resultados tampoco son precisos encontrando algunos estudios que ponen de manifiesto que los docentes con más años de experiencia muestran actitudes más positivas (De Boer et al., 2011; Hind et al., 2019; Vicuña, 2013) pero, por el contrario, otros concluyen que los maestros con más experiencia tienden a tener actitudes más negativas hacia la inclusión (Vaz et al., 2015). En lo que respecta a la interacción con personas con discapacidad, encontramos estudios que relacionan dicha interacción con actitudes más positivas (Dias \& Cadime, 2016; Kwon et al., 2017; Martínez y Bilbao, 2011) y otros por el contrario indican que un contacto frecuente da lugar a actitudes negativas (Muratori et al., 2010).

Tomando como referencia la revisión aportada sobre las actitudes hacia la discapacidad de los docentes y su influencia en la puesta en marcha de un modelo inclusivo es preciso conocer, evaluar y determinar dichas actitudes del profesorado hacia los alumnos con discapacidad y su inclusión. Así se podrá comprobar si los resultados concuerdan con estudios previos y si se han registrado avances en el área temática. Teniendo en cuenta ciertas características de los docentes el objetivo de dicha investigación es conocer si existen diferencias en las actitudes del profesorado (desarrollo social, clima del aula, formación y recursos, rendimiento y responsabilidad) en función del sexo, la edad y si tienen experiencia previa o no trabajando con estudiantes que presentan Necesidades Educativas Especiales (NEE, de aquí en adelante).

\section{Método}

\section{Diseño del Estudio}

El estudio presentado se engloba dentro de los diseños ex post facto ya que el investigador estudia variables sin pretender cambiar los resultados, actúa una vez que ya se han producido los hechos. Como indica Juste, Galán y Díaz (2012) en estos estudios no hay manipulación sobre la variable independiente, el investigador observa o analiza lo que ocurre en su contexto sin intervenir de manera alguna.

\section{Participantes}

La muestra fue seleccionada mediante un muestreo no probabilístico (accesibilidad), siendo la participación voluntaria. Estaba compuesta por 281 docentes (69,4\% eran mujeres y 30,6\% hombres) de centros educativos ordinarios. Estos son centros escolares de Educación Infantil, Primaria o Secundaria que, de acuerdo con el modelo de atención a la diversidad del sistema educativo español, desarrollan medidas para atender a las necesidades educativas contemplando la totalidad del alumnado escolarizado. Los docentes que participaron pertenecían a escuelas ubicadas en zonas urbanas $(70,8 \%)$ y entornos rurales $(28,2 \%)$. Enseñaban en diferentes etapas educativas (preescolar $=18,1 \%$, primaria $=45,6 \%$ y secundaria $=36,3 \%)$, tanto en colegios públicos $(57,7 \%)$ como privados (42,3\%). Los participantes tenían entre 22 y 64 años $\left(X^{-}=38\right.$; DT $\left.=9,09\right)$ y el 89,3\% presentaron experiencia docente previa trabajando con estudiantes que presentaban NEE. 


\section{Instrumentos}

Para evaluar las actitudes de los docentes se ha utilizado el cuestionario de Solís, Pedrosa y Mateos (2019) que es una versión revisada y validada del cuestionario de Doménech et al. (2004) sobre actitudes docentes hacia estudiantes con necesidades educativas especiales. Dicho cuestionario está formado por 22 ítems con un formato de respuesta tipo Likert, en donde 1 implica encontrarse 'Muy en desacuerdo' y 5 'Muy de acuerdo' con la afirmación. A continuación, se especifica sus cinco dimensiones junto con los valores de fiabilidad (Alpha y Omega) obtenidos en este estudio. Responsabilidad (cuatro ítems, $\alpha=0,58$; $\omega=0,30$ ), hace referencia al grado de implicación personal de los docentes (p. ej. Creo que la formación de los alumnos con discapacidad es responsabilidad de los especialistas), Rendimiento (tres ítems, $\alpha=$ $0,42 ; \omega=0,48)$, evalúa la opinión de los docentes sobre cómo la inclusión afecta al rendimiento de los alumnos (p. ej. El reto que representa estar en una clase ordinaria estimula el desarrollo académico del alumno con discapacidad), Formación y recursos (seis ítems, $\alpha=0,78 ; \omega=0,60$ ), se refiere a la capacitación y recursos disponibles para la puesta en marcha de prácticas inclusivas (p. ej. Creo que el centro ofrece la suficiente formación para que los profesores amplíen su conocimiento sobre la discapacidad), Clima del aula (cuatro ítems, $\alpha=0,78, \omega=0,64$ ) relativo a cómo afecta la inclusión a la convivencia en el aula (p. ej. Es difícil mantener el orden y disciplina en una clase ordinaria a la que asisten alumnos con discapacidad) y Desarrollo Social (cinco ítems, $\alpha=0,83 ; \omega=0,71$ ) que incide en como la inclusión implica una evolución en las relaciones de los individuos (p. ej. La imagen social de los alumnos con discapacidad tal y como la ven los compañeros, mejora a través de la interacción con el grupo). En cuanto a la validez del instrumento se muestra un ajuste adecuado $(\mathrm{GFI}=0,93 ; \mathrm{RMSR}=0,06)$.

\section{Procedimiento y Análisis de Datos}

La aplicación del instrumento se realizó online de manera anónima y voluntaria, tras la aprobación de los directores de los diferentes centros seleccionados. Una vez recogidos y organizados los datos, para dar respuesta al objetivo de investigación y estudiar si existen diferencias en las dimensiones de la escala de actitud (desarrollo social, clima del aula, formación y recurso, rendimiento y responsabilidad) en función del sexo, la edad y la experiencia previa trabajando con estudiantes con NEE, siguiendo las recomendaciones de Pardo y San Martín (2010) se comprobó el supuesto de normalidad y el de igualdad de varianzas (homocedasticidad). El primero de ellos mediante los estadísticos Kolmogórov-Smirnov y Shapiro-Will y los gráficos Q-Q; en cuanto al supuesto de homocedasticidad entre las poblaciones, se constató mediante la prueba de Levene (basada en medias). El incumplimiento de dichos supuestos nos llevó, tal y como recomiendan los autores anteriores, a la utilización de pruebas no paramétricas, concretamente a la prueba basada en rangos promedios, U de Mann-Whitney, acompañada de los estadísticos $\mathrm{W}$ de Wilcoxon y $\mathrm{Z}$ (una tipificación de los estadísticos $\mathrm{U}$ y W), ya que contábamos con variables categóricas: sexo (hombre y mujer), experiencia previa trabajando con estudiantes con NEE, categorizada como sí y no y edad codificada como menos de 41 años y 41 años o más. Esta última recodificación se debe a que según Marente y Gestoso (2008) es a partir de los 40 años cuando los docentes se pueden sentir más "quemados" (burnout) con la docencia y, por tanto, afectar a sus actitudes. Finalmente, para conocer la magnitud de las diferencias encontradas, se calculó el tamaño del efecto mediante el estadístico d de Cohen donde un valor comprendido entre 0,20 y 0,49 indica un efecto pequeño, valores entre 0,50 y 0,80 un efecto mediano y valores mayores de 0,80 un efecto grande (Cohen, 1992). Para los análisis, se utilizó el programa estadístico SPSS versión 25.0 (IBM ® SPSS $®$ Statistics 25) y el programa $G^{*}$ Power 3.1 para el cálculo del tamaño del efecto. El nivel de confianza establecido fue del $95 \%(\mathrm{p}<0,05)$.

\section{Resultados}

A continuación, se presentan los resultados referidos a las diferencias existentes en la variable sexo, edad y experiencia trabajando con estudiantes con NEE, en las distintas dimensiones de la escala de actitud (desarrollo social, clima del aula, formación y recursos, rendimiento y responsabilidad).

- Sexo 
Como podemos observar en la tabla 1, los hombres presentan rangos promedios superiores a los de las mujeres en todas las dimensiones, menos en la dimensión de responsabilidad (las mujeres obtienen 11,41 puntos más). Siendo estas diferencias significativas en todos los casos ( $\mathrm{p}<0,05)$, aunque en la dimensión clima social, el valor del estadístico está muy cercano al nivel de riesgo $(0,05)$. En cuanto a los tamaños del efecto siguiendo a Cohen (1992) se consideran pequeños.

Tabla 1.

Descriptivos y prueba de Mann-Whitney para la variable sexo.

\begin{tabular}{cccccccc}
\hline & & & N & $\begin{array}{c}\text { Desarrollo } \\
\text { Social }\end{array}$ & Clima del & Formación y & \\
aula & recursos & Rendimiento Responsabilidad \\
\hline Rango medio & Mujer & 195 & 134.02 & 134.68 & 132.79 & 134.21 & 144.49 \\
& Hombre & 86 & 156.84 & 155.33 & 159.61 & 156.39 & 133.08 \\
U de Mann-Whitney & & & 7023.00 & 7153.00 & 6784.50 & 7703.50 & 6604.50 \\
W de Wilcoxon & & & 26133.00 & 26263.00 & 25894.50 & 11444.50 & 25714.50 \\
Z & & & -2.20 & -1.98 & -2.55 & -1.10 & -2.84 \\
Sig. asintót. (bilateral) & & & .028 & .047 & .011 & .271 & .005 \\
Tamaño del efecto $(d)$ & & & 0.26 & 0.23 & 0.30 & 0.13 & 0.34 \\
\hline
\end{tabular}

- Edad

En cuanto a la variable edad, recodificada como docentes con menos de 41 años y con más de 40 años, los resultados obtenidos en la prueba de U de Mann-Whitney, tal y como se observa en la tabla 2, muestran que los docentes comprendidos entre los 22 y 40 años puntúan más en la dimensión desarrollo social y clima del aula, siendo esta diferencia significativa $(\mathrm{p}<0,05)$ respecto a los docentes que tienen entre 41-64 años. También obtienen una puntuación ligeramente superior en la dimensión de rendimiento, pero no es significativa. En la dimensión de formación y recursos, así como en la de responsabilidad, puntúan más alto los docentes comprendidos entre los 41 y 64 años, siendo no significativa dicha diferencia $(\mathrm{p}>0,05)$ respecto al grupo de docentes comprendidos entre los 22 y 40 años. En cuanto a los tamaños del efecto están próximos a ser medios (Cohen, 1992).

Tabla 2.

Descriptivos y prueba de Mann-Whitney para la variable edad.

\begin{tabular}{|c|c|c|c|c|c|c|c|}
\hline & & $\mathbf{N}$ & $\begin{array}{c}\text { Desarrollo } \\
\text { Social } \\
\end{array}$ & $\begin{array}{c}\text { Clima del } \\
\text { aula }\end{array}$ & $\begin{array}{c}\text { Formación y } \\
\text { recursos }\end{array}$ & Rendimiento & Responsabilidad \\
\hline \multirow[t]{2}{*}{ Rango medio } & 22-40 años & 180 & 154.94 & 151.91 & 137.16 & 141.06 & 135.54 \\
\hline & 41-64 & 101 & 116.15 & 121.55 & 147.84 & 140.89 & 150.74 \\
\hline U de Mann-Whitney & & & 6580.50 & 7126.00 & 8399.50 & 9078.50 & 8106.50 \\
\hline W de Wilcoxon & & & 11731.50 & 12277.00 & 24689.50 & 14229.50 & 24396.50 \\
\hline $\mathrm{Z}$ & & & -3.83 & -3.04 & -1.06 & -.02 & -1.53 \\
\hline $\begin{array}{l}\text { Sig. asintót. } \\
\text { (bilateral) }\end{array}$ & & & .000 & .002 & .290 & .986 & .127 \\
\hline $\begin{array}{c}\text { Tamaño del efecto } \\
(d)\end{array}$ & & & 0.47 & 0.37 & & & \\
\hline
\end{tabular}

- Experiencia previa con estudiantes con NEE

Como se observa en la tabla 3 existen diferencias significativas entre los docentes que han tenido experiencia previa trabajando con estudiantes que tienen NEE y los que no, en las dimensiones de desarrollo social, clima de aula, rendimiento y responsabilidad, puntuando más alto los docentes que no 
han trabajado con estudiantes con NEE, a excepción de la dimensión responsabilidad, ya que las puntuaciones son más altas en los docentes que sí han trabajado con estudiantes con NEE. En cuanto al tamaño del efecto se observan valores entre bajos y moderados (Cohen, 1992).

Tabla 3.

Descriptivos y prueba de Mann-Whitney para la variable experiencia previa con estudiantes con NEE.

\begin{tabular}{|c|c|c|c|c|c|c|c|}
\hline & & $\mathbf{N}$ & $\begin{array}{c}\text { Desarrollo } \\
\text { Social }\end{array}$ & $\begin{array}{c}\text { Clima del } \\
\text { aula }\end{array}$ & $\begin{array}{c}\text { Formación y } \\
\text { recursos }\end{array}$ & Rendimiento & Responsabilidad \\
\hline \multirow[t]{2}{*}{ Rango medio } & Sí & 251 & 134.83 & 132.24 & 139.80 & 133.44 & 144.75 \\
\hline & No & 30 & 192.63 & 214.33 & 151.03 & 204.28 & 109.62 \\
\hline U de Mann-Whitney & & & 2216.00 & 1565.00 & 3464.00 & 1866.50 & 2823.50 \\
\hline W de Wilcoxon & & & 33842.00 & 33191.00 & 35090.00 & 33492.50 & 3288.50 \\
\hline $\mathrm{Z}$ & & & -3.74 & -5.29 & -.72 & -4.56 & -2.27 \\
\hline Sig. asintót. (bilateral) & & & .000 & .000 & .473 & .000 & .023 \\
\hline Tamaño del efecto $(d)$ & & & 0.45 & 0.65 & & 0.56 & 0.27 \\
\hline
\end{tabular}

\section{Discusión}

Los sistemas educativos alrededor de todo el mundo se enfrentan al desafío de lograr la inclusión educativa (Ainscow, 2005), este desafío se aborda lenta y gradualmente (Hagiwara et al., 2019) y los docentes pueden ser un facilitador o bien una barrera (Angenscheidt \& Navarrete, 2017). Por este motivo en el presente estudio se estudian las actitudes de los docentes hacia la discapacidad analizando sus diferencias en función del sexo, la edad y la experiencia previa con alumnos con discapacidad.

A partir de los resultados obtenidos podemos llegar a las siguientes conclusiones, acorde a las variables estudiadas:

\section{- Sexo}

Respecto a la variable sexo se concluye que los hombres presentan rangos promedios superiores a los de las mujeres en casi todas las dimensiones (desarrollo social, clima de aula, formación y recursos y rendimiento) de la escala de actitud, menos en la dimensión de responsabilidad, siendo estas diferencias significativas $(p<0,05)$. Este hecho contradice a algunos estudios previos en los que se deduce que apenas se encuentran diferencias significativas (Hodge et al., 2002; Parasuram, 2006) y en caso de haberlas son las mujeres quienes expresan actitudes más pro-inclusión (Pegalajar \& Colmenero, 2017; Solís \& Borja, 2021; Vaz et al., 2015). Merece la pena profundizar en este dato ya que Novo-Corti et al. (2015) destacan la importancia del sexo como factor diferenciador, más aún, teniendo en cuenta que en el sector profesional en el que nos encontramos hay sin duda un mayor porcentaje de mujeres.

\section{- Edad}

En lo que se refiere a la variable edad, los docentes con edades comprendidas entre los 22 y 40 años puntúan más alto en la dimensión desarrollo social y clima del aula, siendo esta diferencia significativa $(p<0,05)$ respecto a los docentes que tienen entre 41-64 años. Esto es, los docentes más jóvenes consideran que la inclusión favorece la socialización, parten del concepto de inclusión como elemento enriquecedor del aula. Estudios previos han puesto de relieve la importancia de la afectividad como un recurso pedagógico para la comunicación con estudiantes con NEE (Zenteno-Osorio \& LealSoto, 2016). Sin embargo, en la dimensión de formación y recursos, así como en la de responsabilidad puntúan más alto los docentes comprendidos entre los 41 y 64 años, aunque dicha diferencia no es significativa. Este dato contrasta con los datos aportados por Domínguez-Alonso et al. (2015) quienes en un estudio previo encuentran que a mayor edad de los docentes se otorga menos importancia a la atención a la diversidad. Este hecho podría relacionarse con los programas formativos: el profesorado de más edad se ha formado en un modelo de integración frente al modelo inclusivo más reciente. Con respecto a la variable responsabilidad es necesario apuntar que aún existen profesores que creen que la responsabilidad 
de la formación de los estudiantes con NEE recae en el maestro de apoyo o en los especialistas, por ello el peso sigue siendo soportado principalmente por estos últimos (Echeíta \& Fernández, 2017; GonzálezRojas \& Triana-Fierro, 2018). En síntesis, respecto a la variable edad observamos que los profesores más jóvenes tienen una concepción más idealizada acerca de la inclusión de alumnos con discapacidad, sin embargo, los profesores de más edad perciben tener una mayor formación y recursos para afrontar con responsabilidad dicho desafío.

\section{- Experiencia trabajando con estudiantes con NEE}

La experiencia previa docente es un factor que condiciona la actitud del profesorado (De Boer et al., 2011; González \& Macías, 2018). En la variable experiencia trabajando con estudiantes con NEE, se observó que existen diferencias significativas entre los docentes que han tenido experiencia previa trabajando con estudiantes que tienen NEE y los que no, en las dimensiones de desarrollo social, clima de aula, rendimiento y responsabilidad, puntuando más alto los docentes que no han trabajado con estudiantes con NEE, a excepción de la dimensión responsabilidad, ya que las puntuaciones son más altas en los docentes que sí han trabajado con estudiantes con NEE.

Angenscheidt y Navarrete (2017) encuentran que los docentes con más experiencia tienen una actitud más favorable hacia la implementación de prácticas inclusivas. En nuestro caso, el hecho de que los docentes con experiencia previa en el ámbito muestren actitudes menos favorables en cuanto al desarrollo social, clima de aula, rendimiento y responsabilidad puede estar relacionada con una sobrecarga de trabajo (Marente \& Gestoso, 2008) o con un desencanto de medidas inclusivas previas, así se ha constatado el efecto que imprime la cultura escolar sobre las emociones de los docentes (Poblete-Christie et al., 2019).

\section{Conclusiones}

En líneas generales, las actitudes expresadas por la muestra son moderadamente positivas hallándose paralelismo con otros estudios previos (Dias \& Cadime, 2016; Rakap et al., 2016; Saloviita \& Schaffus, 2016; Solís et al., 2019) siendo las actitudes favorables de los docentes un requerimiento para lograr poner en marcha un sistema inclusivo (Saloviita, 2019). Asimismo, se pone de relevancia la importancia del sexo y de la edad como elemento de diferencia en determinadas variables que afectan a la inclusión, siendo necesario profundizar en las mismas. Se constata además que la experiencia previa en el aula con alumnos con discapacidad influye en las actitudes, aunque no siempre favorablemente.

Una vez discutidos los resultados debemos abordar las limitaciones presentes. Una de las limitaciones de esta investigación se debe a que la muestra no ha sido seleccionada al azar, lo que dificulta la generalización de los resultados al resto de la población (validez externa).

Otra limitación metodológica importante, que nos lleva a que los resultados deban ser analizados con cautela es que los valores de fiabilidad encontrados en el instrumento no siempre superan el valor mínimo establecido de 0,70 (Streiner, 2003). También es importante tener en cuenta la diferencia existente entre los tamaños muestrales de las variables: sexo (mujer $=195$; hombre $=86)$, edad $(<41=180 ;\rangle 40=$ 101) y la variable experiencia trabajando con estudiantes con NEE ( $S i ́=251$; No =30), aunque estas discrepancias muestrales hacen que los análisis tengan que ser interpretados con cierta reserva, se garantizó en la medida de lo posible la fiabilidad de los resultados basados en la comparación de grupos utilizando pruebas no paramétricas. Asimismo, para comprobar que la diferencia entre los tamaños grupales no estuviera sesgando los resultados se replicaron los análisis de comparación equiparando los grupos, teniendo en cuenta el tamaño del grupo más pequeño. Las diferencias encontradas entre los grupos fueron las mismas que las obtenidas en los análisis previos realizados con tamaños muestrales no equiparados. Aun así, en un futuro se prevé trabajar con muestras más equilibradas y probabilísticas.

Respecto a la magnitud de las diferencias, se han encontrado tamaños de efectos bajos y moderados. A la hora de interpretarlos hay que ser cautelosos ya que, aunque el tamaño del efecto está destinado a representar un efecto "verdadero" en la población, es importante entender que las estimaciones del tamaño del efecto pueden verse influenciadas por el muestreo y la medición. Cuando las muestras no son aleatorias, como ocurre en esta investigación, se pueden producir estimaciones del tamaño del efecto 
sesgadas que deben interpretarse con precaución (Ferguson, 2009). Asimismo, como indican Pardo y San Martín (2010) las medidas del tamaño del efecto no se tienen que entender como la única respuesta eficaz en el análisis de datos ya que la significación estadística que ofrece un contraste de hipótesis posee una extraordinaria utilidad como argumento para elegir entre hipótesis rivales.

Las coincidencias y disonancias encontradas con estudios previos animan a profundizar en la influencia de las variables sociodemográficas respecto a las actitudes docentes como primer paso para asegurar el éxito de las prácticas inclusivas y la adecuada atención de calidad a todos los alumnos independientemente de sus características y particularidades.

Contribución de cada utor: conceptualización, P.S.G. y D.A.R.; metodología, D.A.R; análisis, D.A.R.; escritura del manuscrito, P.S.G.; escritura, revision y edición, P.S.G. y D.A.R.; supervisión, P.S.G. y D.A.R.

Financiación: Esta investigación no recibió ningurna financiación externa.

Agradecimientos: En esta sección se pueden escribir los agradecimientos.

Conflicto de Intereses: Las autoras declaran no tener ningún conflicto de intereses.

\section{Referencias}

Ainscow, M. (2005). Developing inclusive education systems: what are the levers for change? Journal of educational change, 6(2), 109-124. https://doi.org/10.1007/s10833-005-1298-4

Allport, G.W. (1935). Attitudes. En C. Murchison (Ed): A handbook of social psychology (pp.798-844). Clark University Press.

Amor, A. M., Hagiwara, M., Shogren, K. A., Thompson, J. R., Verdugo, M. Á., Burke, K. M., \& Aguayo, V. (2019). International perspectives and trends in research on inclusive education: a systematic review. International Journal of Inclusive Education, 23(12), 1277-1295. https://doi.org/10.1080/13603116.2018.1445304

Angenscheidt, L. \& Navarrete, I. (2017). Actitudes de los docentes acerca de la educación inclusiva. Ciencias Psicológicas, 11(2), 233-243. https://doi.org/10.22235/cp.v11i2.1500

Cagran, B. \& Schmidt, M. (2011). Attitudes od Slovene teachers towards the inclusión of pupils with different types of special needs in primary school. Educational Studies, 37, 171-195. https://doi.org/10.1080/03055698.2010.506319

Castillo, N. M. (2019). Educación Inclusiva: Contradicciones, debates y resistencias. Praxis Educativa, 23(3), 29. https://doi.org/10.19137/praxiseducativa-2019-230304

Cruz-Ortiz, M., Pérez-Rodríguez, M. C., Jenaro-Río, C., Sevilla-Santo, D., \& Cruz-Ortiz, S. (2016). Whendifferences do notmatter: Inclusion in a Mexicanprimaryschool. Cultura y Educación, 28, 72-98. https://doi.org/10.1080/11356405.2015.1124549

Cohen, J. (1992). A power primer. Psychological Bulletin, 112, 155-159. https://doi.org/10.1037/00332909.112.1.155

De Boer, A., Pijl, S. J., \& Minnaert, A. (2011). Regular primary schoolteachers' attitudes towards inclusive education: A review of the literature. International Journal of Inclusive Education, 1, 1-24. https://doi.org/10.1080/13603110903030089

Dias, P. C. \& Cadime, I. (2016). Effects of personal and professional factors on teachers' attitudes towards inclusion in preschool. European Journal of Special Needs Education, 31(1), 111-123. https://doi.org/10.1080/08856257.2015.1108040

Doménech, V., Esbrí, J. V., González, H. A., \& Miret, L. (2004). Actitudes del profesorado hacia el alumnado con necesidades educativas especiales derivadas de discapacidad. España: Novenes Jornades de Foment de la Investigació, Universitat Jaume I, Castelló.

Domínguez-Alonso, J., López-Castedo, A., Pino, M. R., \& Vázquez, E. (2015). Integración o inclusión: El dilema educativo en la atención a la diversidad. Revista Portuguesa de Educação, 28(2), 31-50. https://doi.org/10.21814/rpe.7749 
Actitudes docentes hacia alumnos con discapacidad, punto de partida para la educación inclusiva: una perspectiva de sexo, edad y experiencia previa

Echeíta, G. \& Fernández, M. L. (2017). El contexto educativo. Hacia una educación más inclusiva como contexto para el desarrollo de todo el alumnado. En B. Bermejo y Á. Brioso (Coords.), Desarrollos Diferentes (pp. 201-215). Sanz y Torres.

Ferguson, C. J. (2009). An effect size primer: a guide for clinicians and researchers. Professional Psychology: Research and Practice, 40(5), 532-538. https://doi.org/10.1037/a0015808

Fishbein, M. (Ed.). (1967). Readings in attitude theory and measurement. Wiley.

Florian, L. (2019). On the necessary co-existence of special and inclusive education. International Journal of Inclusive Education, 23(7-8), 691-704. https://doi.org/10.1080/13603116.2019.1622801

Gal, E., Schreur, N., \& Engel-Yeger, B. (2010). Inclusion of children with disabilities: Teachers' attitudes and requirements for environmental accommodations. International Journal of Special Education, 25(2), 8999.

Garrad, T. A., Rayner, C., \& Pedersen, S. (2019). Attitudes of Australian primary school teachers towards the inclusion of students with autism spectrum disorders. Journal of Research in Special Educational Needs, 19(1), 58-67. https://doi.org/10.1111/1471-3802.12424

Garzón, P., Calvo, M. I., \& Orgaz, B. (2016). Inclusión educativa. Actitudes y estrategias del profesorado. Revista Española de Discapacidad (REDIS), 4(2), 25-45. https://doi.org/10.5569/2340-5104.04.02.02

González, I. \& Macías, D. (2018). La formación permanente como herramienta para mejorar la intervención del maestro de educación física con alumnado con discapacidad. Retos: Nuevas tendencias en Educación Física, Deportes y Recreación, 33, 118-122. https://recyt.fecyt.es/index.php/retos/article/view/55056

González-Rojas, Y. \& Triana-Fierro, D. A. (2018). Actitudes de los docentes frente a la inclusión de estudiantes con necesidades educativas especiales. Educación y Educadores, 21(2), 200-218. https://doi.org/10.5294/edu.2018.21.2.2

Granada, M., Pomés, M. P. \& Sanhueza, S. (2013). Actitudes de los profesores hacia la inclusión educativa. Papeles de Trabajo, 25, 51-59.

Hagiwara, M., Shogren, K. A., Thompson, J. R., Burke, K. M., Uyanik, H., Amor, A. M., ... \& Aguayo, V. (2019). International trends in inclusive education intervention research: A literature review. Education and Training in Autism and Developmental Disabilities, 54(1), 3-17.

Hall, J. (2018). Integration, Inclusion-What does it all mean? In Whose Choice? (pp. 82-102). Routledge.

Hind, K., Larkin, R., \& Dunn, A. K. (2019). Assessing teacher opinion on the inclusion of children with social, emotional and behavioural difficulties into mainstream school classes. International Journal of Disability, Development and Education, 66(4), 424-437. https://doi.org/10.1080/1034912X.2018.1460462

Hodge, S. R., Davis, R., Woodard, R., \& Sherrill, C. (2002). Comparison of practicum types in changing preservice teachers' attitudes and perceived competence. Adapted Physical Activity Quarterly, 19(2), 155172. https://doi.org/10.1123/apaq.19.2.155

Juste, R. P., González, A. G., \& Díaz, J. Q. (2012). Métodos y diseños de investigación en educación. Editorial UNED

Kwon, K., Hong, S. Y., \& Jeon, H. J. (2017). Classroom readiness for successful inclusion: Teacher factors and preschool children's experience with and attitudes toward peers with disabilities. Journal of Research in Childhood Education, 31, 360-378. https://doi.org/10.1080/02568543.2017.1309480

Kurniawati, F., De Boer, A. A., Minnaert, A. E. M. G., \& Mangunsong, F. (2017). Evaluating the effect of a teacher training programme on the primary teachers' attitudes, knowledge and teaching strategies regarding special educational needs. Educational Psychology, 37(3), 287-297. https://doi.org/10.1080/01443410.2016.1176125

Lindqvist, B. (1994). Información sobre Educación Inclusiva. UN Rapporteur.

Marente, J. A. A., \& Gestoso, C. L. G. (2008). Burnout y mobbing en enseñanza secundaria. Revista complutense de Educación, 19(1), 157.

Martínez, M. A., \& Bilbao, M. C. (2011). Los docentes de la universidad de Burgos y su actitud hacia las personas con discapacidad. Revista Española sobre Discapacidad Intelectual, 42, 50-78. Recuperado de http://sid.usal.es/idocs/F8/ART19441/240-8\% 20Mart\%C3\%ADnez.pdf

Muratori, M., Guntín, C., \& Delfino, G. (2010). Actitudes de los adolescentes hacia las personas con discapacidad: Un estudio con alumnos del polimodal en la zona norte del conurbano bonaerense [en línea]. Revista de

Espiral. Cuadernos del Profesorado | ISSN 1988-7701 | 2022, 15(30), 72-81 
Psicología, 6(12), 39-56. Recuperado de http://bibliotecadigital.uca.edu.ar/repositorio/revistas/actitudesadolescentes-hacia-personas-discapacidad.pdf

Novo-Corti, I., Muñoz-Cantero, J. M., \& Calvo-Babío, N. (2015). Los futuros docentes y su actitud hacia la inclusión de personas con discapacidad. Una perspectiva de género. Anales de Psicología, 31(1), 155171. https://doi.org/10.6018/analesps.31.1.163631

OCDE. (2013). Panorama de la educación: Indicadores de la OCDE 2013 (Informe español). Recuperado de: http://www.mecd.gob.es/dctm/inee/internacional/panoramadelaeducacion2013informeespanol.pdf?documentId=0901e72b816996b6

Parasuram, K. (2006). Variables that affect teachers' attitudes towards disability and inclusive education in Mumbai, India. Disability \& Society, 21, 231-242. https://doi.org/10.1080/09687590600617352

Pardo, A. y San Martín, R, (2010). Análisis de datos en Ciencias Sociales y de la Salud II. Editorial Síntesis.

Pegalajar, M. D. C., \& Colmenero, M. D. J. (2017). Actitudes y formación docente hacia la inclusión en Educación Secundaria Obligatoria. Revista Electrónica de Investigación Educativa, 19(1), 84-97. https://doi.org/10.24320/redie.2017.19.1.765

Pérez-Jorge, D., Alegre, O. M., Rodríguez-Jiménez, M.C, Márquez-Domínguez, Y., \& de la Rosa, M. (2016). La identificación del conocimiento y actitudes del profesorado hacia inclusión de los alumnos con Necesidades Educativas Especiales. European Scientific Journal, 12(7). https://doi.org/10.19044/esj.2016.v12n7p64

Poblete-Christie, O., López, M., \& Muñoz, L. (2019). ¿De la frustración a la alegría o de la alegría a la frustración? Significados sobre inclusión educativa construidos por docentes a partir de su experiencia emocional. Psykhe (Santiago), 28(1), 1-14. https://doi.org/10.7764/psykhe.28.1.1126

Rakap, S., Parlak-Rakap, A., \& Aydin, B. (2016). Investigation and comparison of Turkish and American preschool teacher candidates' attitudes towards inclusion of young children with disabilities. International Journal of Inclusive Education, 20(11), 1223-1237. https://doi.org/10.1080/13603116.2016.1159254

Rodríguez, M. (2015). Actitudes hacia la discapacidad en alumnos de Magisterio de Educación Infantil. Propuestas de formación para una Educación Inclusiva. Revista de Educación Inclusiva, 8(3).

Saloviita, T. (2019). Teacher attitudes towards the inclusion of students with support needs. Journal of Research in Special Educational Needs. https://doi.org/10.1111/1471-3802.12466

Shakespeare, T. (2006). The social model of disability. The Disability Studies Reader, 2, 197-204.

Solís, P., \& Borja, V. (2021). Actitudes del profesorado de Educación Física hacia la inclusión de alumnos con discapacidad. Retos, 39, 7-12. https://doi.org/10.47197/retos.v0i39.77841

Solís, P., Pedrosa, I., \& Mateos, L. M. (2019). Evaluación e interpretación de la actitud del profesorado hacia alumnos con discapacidad. Cultura y Educación, Culture and Education, 31(3), 589-604. https://doi.org/10.1080/11356405.2019.1630955

Streiner, D. L. (2003). Starting at the beginning: an introduction to coefficient alpha and internal consistency. Journal of personality assessment, 80(1), 99-103. https://doi.org/10.1207/S15327752JPA8001_18

Triandis, H. C. (1971). Attitude and attitude change (Vol. 8). Wiley.

UNESCO. (2009). Directrices políticas sobre inclusión en educación. UNESCO.

Vaz, S., Wilson, N., Falkmer, M., Sim, A., Scott, M., Cordier, R., \& Falkmer, T. (2015). Factors associated with primary school teachers' attitudes towards the inclusion of students with disabilities. PloS one, 10(8), https://doi.org/10.1371/journal.pone.0137002

Vicuña, K. D. (2013). Percepciones y actitudes de profesores de primero a séptimo año de Educación General Básica del Distrito Educativo 4 hacia la inclusión del alumnado con necesidades educativas especiales como indicadores de uno de prácticas educativas inclusivas en el aula (Masters' Thesis). Universidad Casa Grande, Ecuador.

Zenteno-Osorio, S., \& Leal-Soto, F. (2016). Los afectos en la experiencia de ser profesor de un estudiante diagnosticado con trastorno espectro autista. Pensamiento Educativo, 53(1), 1-14. https://doi.org/10.7764/PEL.53.1.2016.5 